\title{
Respiratory Distress and Meconium Aspiration Syndrome: Effect of Neonatal Resuscitation Program (NRP) in Ilam, Iran
}

\author{
Sajad Norollahi (iD) ${ }^{1}$, Aliashraf Mozafari ${ }^{2}$, Golnaz Azami (iD ${ }^{3}$ and Elham Shafiei ${ }^{4,}$ \\ ${ }^{1}$ Division of Neonatology, Department of Pediatrics, Ayatollah Taleghani Hospital, Ilam University of Medical Sciences, Ilam, Iran \\ ${ }^{2}$ Department of Epidemiology and Biostatistics, Tehran University of Medical Sciences, Tehran, Iran \\ ${ }^{3}$ Depratment of Nursing and Midwifery, Ilam University of Medical Sciences, Ilam, Iran \\ ${ }^{4}$ Clinical Research Development Unit, Shahid Mostafa Khomeini Hospital, Ilam University of Medical Sciences, Ilam, Iran \\ "Corresponding author: Clinical Research Development Unit, Shahid Mostafa Khomeini Hospital, Ilam University of Medical Sciences, Ilam, Iran. Email: \\ shafiei-e@medilam.ac.ir
}

Received 2020 October 06; Revised 2021 March 07; Accepted 2021 April 10.

Keywords: Meconium Aspiration Syndrome, Neonatal Resuscitation Program

\section{Dear editor,}

Meconium aspiration syndrome (MAS) is a leading cause of neonatal morbidity and mortality. MAS is defined as respiratory distress in the first four hours after birth in the presence of meconium-stained amniotic fluid (MSAF) (1). MAS is categorized as moderate (requiring oxygen therapy with a fraction of inspired oxygen $\left(\mathrm{FIO}_{2}\right)$ of $40 \%$ or greater for at least $24 \mathrm{~h}$ ) or severe (requiring assisted mechanical ventilation) (2). MAS can lead to the development of pulmonary injuries by a different mechanism (3). MSAF may contribute to multiple long-term and short-term adverse outcomes, including increased risk of neonatal resuscitation, low Apgar score, respiratory distress, pulmonary diseases, and neonatal sepsis. Globally, the incidence of MAS is reported to be $5 \%-10 \%$ in infants, which accounts for an estimated $12 \%$ of all neonatal deaths (4). Over the past several years, there have been tremendous advancements in the treatment of MAS. The Neonatal Resuscitation program (NRP) has found widespread acceptance globally as a practical tool to improve newborn survival and reduce the risk and complications of MAS (5). The global implementation of NRP began on January 2, 2016 (6), aiming at providing the cognitive, technical, and behavioral skills needed to successfully resuscitate neonates after delivery (7). This study was conducted at two primary and secondary teaching hospitals in Ilam city, Iran, using a cross-sectional design during 2015 - 2017. A purposive sample of 57 neonates with MAS was recruited for the study. Non-vigorous newborns were included in this study through MSAF. Excluded neonates were those with insufficient information in medical records, intrauterine death, out-of-hospital birth, congenital heart disease, congenital anomalies, maternal disease during pregnancy, and complications during birth. The participants were divided into two groups conveniently. Groups I and II received usual care $(\mathrm{n}=24)$ and NRP protocol $(\mathrm{n}=33)$, respectively. There were significant differences in the length of hospital stay, Apgar score, days on mechanical ventilation, rate of neonatal mortality, and amniotic fluid concentration $(\mathrm{P}<0.05)$. Those who received the NRP protocol had a shorter hospital stay, lower Apgar score, shorter duration of mechanical ventilation, lower rate of mortality, and decreased amniotic fluid concentration than those who received usual care. This pilot study showed promising results on the effectiveness of the NRP protocol. To date, very few studies have been conducted on this topic. Future randomized controlled trials with larger sample sizes and longer follow-up periods are needed to provide more precise evidence.

\section{Footnotes}

Authors' Contribution: Sajad Norollahi, Elham Shafiei, and Aliashraf Mozafari participated in the study conception and design, supervised the study, and revised the manuscript critically for important intellectual content. Elham Shafiei revised the manuscript critically for important intellectual content. All authors read and approved the final manuscript.

Conflict of Interests: The authors have no competing interests to declare.

Funding/Support: It was not declared by the authors. 


\section{References}

1. Paudel P, Sunny AK, Poudel PG, Gurung R, Gurung A, Bastola R, et al. Meconium aspiration syndrome: incidence, associated risk factors and outcome-evidence from a multicentric study in lowresource settings in Nepal. J Paediatr Child Health. 2020;56(4):630-5. doi: 10.1111/jpc.14703. [PubMed: 31894896].

2. Hao LX, Wang F. Effectiveness of high-frequency oscillatory ventilation for the treatment of neonatal meconium aspiration syndrome. Medicine (Baltimore). 2019;98(43). e17622. doi: 10.1097/MD.0000000000017622. [PubMed: 31651876]. [PubMed Central: PMC6824716].

3. Kalra VK, Lee HC, Sie L, Ratnasiri AW, Underwood MA, Lakshminrusimha $S$. Change in neonatal resuscitation guidelines and trends in incidence of meconium aspiration syndrome in California.JPerinatol. 2020;40(1):46-55. doi: 10.1038/s41372-019-0529-0. [PubMed: 31611615].
4. Thornton PD, Campbell RT, Mogos MF, Klima CS, Parsson J, Strid M. Meconium aspiration syndrome: Incidence and outcomes using discharge data. Early Hum Dev. 2019;136:21-6. doi 10.1016/j.earlhumdev.2019.06.011. [PubMed: 31295648].

5. Aldhafeeri FM, Aldhafiri FM, Bamehriz M, Al-Wassia H. Have the 2015 Neonatal Resuscitation Program Guidelines changed the management and outcome of infants born through meconium-stained amniotic fluid? Ann Saudi Med. 2019;39(2):87-91. doi: 10.5144/02564947.2019.87. [PubMed: 30955017]. [PubMed Central: PMC6464672].

6. Zaichkin JG. Neonatal Resuscitation: Neonatal Resuscitation Program 7th Edition Practice Integration. Crit Care Nurs Clin North Am. 2018;30(4):533-47. doi: 10.1016/j.cnc.2018.07.009. [PubMed:30447812].

7. Neonatal Resuscitation Program Steering C. Response From the Neonatal Resuscitation Program (NRP) Steering Committee. Pediatrics. 2019;143(3). doi: 10.1542/peds.2018-4022A. [PubMed: 30819973]. 